\title{
The role of a single olfactory receptor in odorant perception: relating receptor space to perceptual space
}

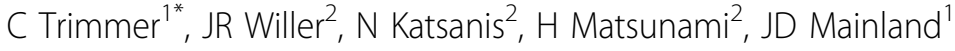 \\ From 1st International Workshop on Odor Spaces \\ Hannover, Germany. 4-7 September 2013
}

\begin{abstract}
Although odorant identity is encoded by the activation patterns of olfactory receptors, we do not know how alterations in receptor activation affect the perceptual properties of an odorant. Human beings display considerable variation in odor perception, very little of which has been concretely linked to molecular causes. Here, we take advantage of genetic variations that affect human odorant receptor function to study the resulting alterations in olfactory perception. Based on previous studies demonstrating that amino acid alterations in OR7D4 affect perception of the odorant androstenone, we hypothesized that subjects with a nonfunctional odorant receptor allele will display varied perception of the receptor's ligand, specifically a reduction in intensity and an increase in valence, in comparison to subjects with functional alleles. To address this issue, we asked 321 human subjects to rate the intensity and valence of 68 odors. We then developed a protocol to sequence each subject's OR subgenome with high coverage. In a genotype/phenotype association analysis, we identified 27 polymorphisms in 8 distinct gene clusters that are associated with alterations in odorant perception more strongly than the association of the OR7D4 $\mathrm{R} 88 \mathrm{~W}$ variant with androstenone intensity in the same data set. Additionally, 35 polymorphisms fall above the 5\% false discovery rate cutoff calculated by permutation analysis. These polymorphisms are found in 11 distinct olfactory receptor gene clusters, but extensive linkage disequilibrium precludes the identification of a single causal receptor. To address this problem, we generated clones of all major receptor variants found in a cluster on chromosome 11 containing 8 odorant receptor genes. These variants were subsequently tested in a high-throughput
\end{abstract}

luciferase assay optimized for the study of olfactory receptor activation. We found that an OR10G4 allele associated with a significant decrease in the perceived intensity of guaiacol, A9V/M134V/V195E/R235G/K265Q, displayed a greatly reduced ability to respond to this odorant in vitro, suggesting a mechanistic basis for the genotype/phenotype association and linking perceptual variation to alterations in receptor function. This work suggests that despite the combinatorial nature of the olfactory code, alterations in a single odorant receptor can have a significant effect on odor perception and is a first step toward the development of a model relating receptor activation and odor perception.

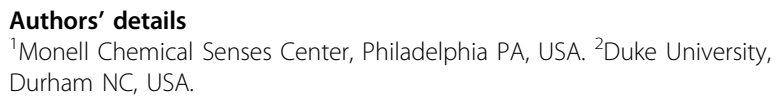

Published: 16 April 2014

doi:10.1186/2044-7248-3-S1-P20

Cite this article as: Trimmer et al:: The role of a single olfactory receptor in odorant perception: relating receptor space to perceptual space. Flavour 2014 3(Suppl 1):P20.

${ }^{1}$ Monell Chemical Senses Center, Philadelphia PA, USA

Full list of author information is available at the end of the article 